\title{
No eczema without keratinocyte death
}

\author{
Thomas Schwarz
}

See related article,

pages 25-35.

Ludwig Boltzmann Institute for Cell Biology and Immunobiology of the Skin, Department of Dermatology,

University Münster, Von-Esmarchstrasse 56, D-48149 Münster, Germany.

Phone: 49-251-8356565; Fax: 49-251-8358579; E-mail: schwtho@uni-muenster.de.

Eczematous dermatitis comprises a heterogenous group of inflammatory skin diseases characterized by a typical morphological reaction pattern. In the acute stage, the typical clinical features include itching, redness, papules, and vesicles associated with exudation. The two most frequent reasons for an eczematous reaction are atopic dermatitis (AD) and allergic contact dermatitis (ACD). Although $\mathrm{AD}$ and $\mathrm{ACD}$ are completely different in nature and pathogenetically unrelated, both disorders in the acute stage share common morphological features, especially vesicle formation, which ultimately causes a disruption of the epidermal barrier. The loss of this protective shield allows additional external noxious agents of microbial, chemical, or physical origin to enter the skin and to aggravate and to perpetuate the disease. Hence, vesicle formation and subsequent loss of the epidermal barrier is a key event in acute eczematous reactions.

Histopathologically, eczematous reactions are characterized by a mononuclear infiltrate with an intercellular epidermal edema, a condition called spongiosis. Severe spongiosis causes keratinocytes' intercellular attachments to rupture and is regarded as the major culprit in generating vesicles in acute eczematous dermatitis (1). In this issue of the JCI, however, Trautmann et al. (2) provide evidence that another pathway, induction of apoptosis of keratinocytes via activation of the Fas receptor system, also disrupts the epidermal barrier, and they argue that infiltrating $\mathrm{T}$ lymphocytes are the direct cause of keratinocyte death through this novel pathway.

Cell death by apoptosis is a tightly regulated process that enables removal of unnecessary, aged, or damaged cells. During apoptosis a complex death program becomes initiated that ultimately leads to the fragmentation of the cell, finally breaking it up into membrane-enclosed bodies that are phagocytosed by macrophages. Because it does not involve the release of inflammatory mediators, apoptosis, in sharp contrast to necrosis, does not typically provoke an inflammatory reaction. Nevertheless, inflammation can induce local apoptosis, and apoptosis-associated inflammation is seen in such conditions as viral hepatitis and Hashimoto's thyroiditis, as well as eczematous dermatitis.

One way to induce apoptosis is by triggering a family of transmembrane proteins called death receptors (3), of which the Fas protein (CD95) may be the most important (4), since Fasinduced apoptosis appears to be

\section{Secretion of IFN- $\gamma$ by T lymphocytes promotes Fas upregulation in keratinocytes, a crucial early step in the apoptotic pathway. Keratinocyte apoptosis only occurs in association with an inflammatory reaction, but the inflammatory infiltrate is the cause, not the consequence, of apoptosis}

involved in a variety of diseases, including Hashimoto's thyroiditis and Helicobacter pylori-induced gastritis $(5,6)$. Triggering of Fas either by agonistic antibodies or by its cognate ligand FasL induces apoptosis. Ligand binding causes trimerization of Fas, and the trimerized cytoplasmic region transduces the signal by recruiting the adapter molecule FADD (Fas-associating protein with death domain). FADD is responsible for downstream signal transduction by recruitment of the cysteine protease, caspase-8. Subsequently, a cascade of downstream caspases executes apoptotic cell death (4).

Perhaps the best studied of the cells subject to this apoptotic pathway are lymphocytes. Fas and its ligand are essential for normal homeostasis of the lymphoid system, as seen when defects in the Fas system result in lymphadenopathy, splenomegaly, or autoimmunity (7). This pathway is also implicated in the progression of tumors, since tumor cells that express high levels of FasL have been shown to escape an immune response by killing Fas-bearing lymphocytes (8). The paper by Trautmann et al. (2) now identifies the keratinocyte as an additional target of Fas-induced apoptosis and provides evidence that this form of cell death contributes to the pathogenesis of eczematous dermatitis. Keratinocytes normally express low levels of Fas, but IFN- $\gamma$ upregulates Fas on these cells $(9,10)$. The authors show that cultured keratinocytes are driven into apoptosis upon coincubation with autologous activated $\mathrm{T}$ lymphocytes. Addition of a blocking Fas-Fc fragment inhibited apoptosis, indicating that keratinocyte death is due to triggering of the Fas system. Secretion of IFN- $\gamma$ by T lymphocytes, which promotes Fas upregulation in keratinocytes, is a crucial early step in this pathway. Therefore, in this case keratinocyte apoptosis occurs only in association with an inflammatory reaction; but it is important to mention that the inflammatory infiltrate is not the consequence but the cause of apoptosis. A similar mechanism may apply to hepatitis B virus-related liver cirrhosis, where $\mathrm{T}$ lymphocytes kill hepatocytes using the Fas system (11). In Hashimoto's thyroiditis, the inflammatory mediator IL- $1 \beta$ seems to be the initiator of thyrocyte death by upregulating Fas on these cells (5).

Fas-mediated keratinocyte death recently has been shown to be involved in another dermatosis, toxic epidermal necrolysis (TEN) (12). TEN is a lifethreatening drug-induced cutaneous reaction with extensive epidermal destruction. Unlike in AD and ACD, in TEN keratinocytes kill themselves by 
expressing FasL and thus do not need the help of lymphocytes. Although the mechanism for upregulation of the killer ligand is unknown, inhibition of the Fas pathway by application of neutralizing Fas antibodies appears to be a promising therapeutic approach (12).

\section{Clinical implications of keratinocyte apoptosis}

From the pathophysiologic point of view, it is interesting that the same mechanism is demonstrated in $\mathrm{AD}$ and $\mathrm{ACD}$, since these dermatoses are usually regarded as mutually exclusive, $\mathrm{AD}$ being a classic example of a T-helper 2-mediated (Th2-mediated) and ACD of a Th1-mediated process (13). Th2 cells preferentially release IL-4, IL-5, and IL-10 but not IFN- $\gamma$. Since IFN- $\gamma$ promotes a Th1 response, IFN- $\gamma$ has even been applied therapeutically in AD, unfortunately not with great success (14). However, Grewe et al. recently suggested that Th 2 cells are important only in the initial stage of $\mathrm{AD}$ and that a shift toward a Th1 response occurs later and leads to high expression of IFN- $\boldsymbol{\gamma}$ in lesions (15). These authors speculated that IFN- $\gamma$ expression via upregulation of the adhesion molecule ICAM-1 contributes to the subsequent accumulation of inflammatory cells. Consistent with this suggestion, the present findings (2) imply that the high expression of IFN- $\gamma$ also propagates the inflammatory process via disruption of the epidermal barrier. Interestingly, in histologic sections of AD and ACD lesions alike, the majority of apoptotic keratinocytes were found not in spongiotic regions, but in areas that retained normal cohesion of epidermal cells. Hence, one can speculate that apoptosis precedes spongiosis and, further, that apoptotic death of keratinocytes pro- motes spongiosis by enabling the influx of extracellular fluid into the epidermis. ACD has always been regarded as a Th1-driven immune response, and the crucial role of the Th1 cytokine IFN- $\gamma$, especially during the induction (sensitization) phase, remains undisputed (16). The findings of Trautmann et al. (2) indicate an additional role for this cytokine in inducing keratinocyte cell death during the manifestation (elicitation) phase of this disease. Interestingly, ACD responds favorably to phototherapy with ultraviolet-B radiation (UVB). Although the mechanism remains unclear, we have observed that UVB can block the biological effects of IFN- $\gamma$ by inhibiting the phosphorylation of the STAT1 protein (17). STAT1, which is critically involved in the signal transduction pathway of IFN- $\gamma$, must be tyrosine-phosphorylated to become active. In light of the present findings (2) that IFN- $\gamma$ is crucial for manifesting ACD, it may be that UVB improves ACD through its inhibitory effect on IFN- $\gamma$ signaling.

Another established treatment for both AD and ACD is the application of immunosuppressive drugs, such as steroids or cyclosporin A, which primarily target inflammatory cells (14). These drugs are very effective but are associated with side effects, so there is great demand for alternative therapeutic concepts. Here, too, the observations of Trautmann et al. (2) may be of value. One of the major factors perpetuating eczematous dermatitis is the disruption of the epidermal barrier. If, as proposed, Fas-mediated apoptosis of keratinocytes is the key event in this process, pharmacologic inhibitors of apoptosis should be of benefit. Such drugs would also be promising candidates to treat other disorders involving excess apoptosis, such as Alzheimer's disease, cerebral stroke, and acute hepatic failure.

1. Burton, J.L. 1992. Eczema, lichenification, prurigo and erythroderma. In Textbook of dermatology. 5th edition. R.H. Champion, J.L. Burton, and F.J.G. Ebling, editors. Blackwell Scientific Press. Oxford, United Kingdom. 537-588.

2. Trautmann, A., et al. 2000. T cell-mediated Fasinduced keratinocyte apoptosis plays a key pathogenetic role in eczematous dermatitis. J. Clin. Invest. 106:25-35 (2000)

3. Ashkenazi, A., and Dixit, V.M. 1998. Death receptors: signaling and modulation. Science. 281:1305-1308.

4. Peter, M.E., and Krammer, P.H. 1998. Mechanisms of CD95(APO-1/Fas)-mediated apoptosis. Curr. Opin. Immunol. 10:545-551.

5. Giordano, C., et al. 1997. Potential involvement of Fas and its ligand in the pathogenesis of Hashimoto's thyroiditis. Science. 275:960-963.

6. Rudi, J. et al. 1998. Involvement of the CD95 (APO-1/Fas) receptor and ligand system in Helicobacter pylori-induced gastric epithelial apoptosis. J. Clin. Invest. 102:1506-1514.

7. Nagata, S., and Suda, T. 1995. Fas and Fas ligand: lpr and gld mutations. Immunol. Today. 16:39-43.

8. Hahne, M., et al. 1996. Melanoma cell expression of Fas (Apo-1/CD95) ligand: implication for tumor immune escape. Science. 274:1363-1366.

9. Matsue, H., Kobayashi, H., Hosokawa, T., Akitaya, T., and Ohkawara, A. 1995. Keratinocytes constitutively express the Fas antigen that mediates apoptosis in IFN- $\gamma$ cultured keratinocytes. Arch. Dermatol. Res. 287:315-320.

10. Sayama, K., Yonehara, S., Watanabe, Y., and Miki, Y. 1994. Expression of Fas antigen on keratinocytes in vivo and induction of apoptosis in cultured keratinocytes. I. Invest. Dermatol. 103:330-334.

11. Galle, P.R., et al. 1995. Involvement of the CD95 (APO-1/Fas) receptor and ligand in liver damage. J. Exp. Med. 182:1223-1230.

12. Viard, I., et al. 1998. Inhibition of toxic epidermal necrolysis by blockade of CD95 with human intravenous immunoglobulin. Science. 282:490-493.

13. Romagnani, S. 1997. The Th1/Th2 paradigm. Immunol. Today. 18:263-266.

14. Brehler, R., Hildebrand, A., and Luger, T.A. 1997. Recent developments in the treatment of atopic dermatitis. J. Am. Acad. Dermatol. 36:983-994.

15. Grewe, M., Gyufko, K., Schöpf, E., and Krutmann, J. 1994. Lesional expression of interferon- $\gamma$ in atopic eczema. Lancet. 343:25-26.

16. Grabbe, S., and Schwarz, T. 1998. Immunoregulatory mechanisms involved in elicitation of allergic contact hypersensitivity. Immunol. Today. 19:37-44.

17. Aragane, Y., Kulms, D., Luger, T.A., and Schwarz, T. 1997. Downregulation of interferon- $\gamma$-activated STAT1 by ultraviolet light. Proc. Natl. Acad. Sci. USA. 94:11490-11495. 